\title{
Trends in socio-economic inequalities in the Scottish diet: 2001-2009
}

\author{
Karen L Barton ${ }^{1, *}$, Wendy L Wrieden ${ }^{2,3}$, Andrea Sherriff ${ }^{4}$, Julie Armstrong ${ }^{5}$ and \\ Annie S Anderson ${ }^{1}$ \\ ${ }^{1}$ Centre for Public Health Nutrition Research, University of Dundee, Ninewells Hospital and Medical School, Dundee \\ DD1 9SY, UK: ${ }^{2}$ School of Pharmacy and Life Sciences, Robert Gordon University, Aberdeen, UK: ${ }^{3}$ Human Nutrition \\ Research Centre, Newcastle University, Newcastle upon Tyne, UK: ${ }^{4}$ University of Glasgow Dental School, Glasgow, \\ UK: ${ }^{5}$ School of Life Sciences, Glasgow Caledonian University, Glasgow, UK
}

Submitted 13 June 2014: Final revision received 5 December 2014: Accepted 7 January 2015: First published online 16 March 2015

\begin{abstract}
Objective: To explore the association between diet and socio-economic position for 2007-2009 and investigate trends in socio-economic inequalities in the Scottish diet between 2001 and 2009 .

Design: UK food purchase data (collected annually from 2001 to 2009) were used to estimate household-level consumption data. Population mean food consumption, nutrient intakes and energy density were estimated by quintiles of an area-based index of multiple deprivation. Food and nutrient intakes estimated were those targeted for change in Scotland and others indicative of diet quality. The slope and relative indices of inequality were used to assess trends in inequalities in consumption over time.

Setting: Scotland.

Subjects: Scottish households ( $n$ 5020).

Results: Daily consumption of fruit and vegetables (200 g, $348 \mathrm{~g}$ ), brown/ wholemeal bread (17 g, 26.5 g), breakfast cereals (16 g, $27 \mathrm{~g})$ and oil-rich (21 g, $40 \mathrm{~g})$ and white fish $(77 \mathrm{~g}, 112 \mathrm{~g})$ were lowest, and that of total bread highest (105 g, 91.5 g) in the most deprived compared with the least deprived households, respectively, for the period 2007-2009. With regard to nutrients, there was no association between deprivation and the percentage of food energy from total fat and saturated fat; however, non-milk extrinsic sugar intakes $(15.5 \%, 14.3 \%)$ and energy density $(741 \mathrm{~kJ} / 100 \mathrm{~g}, 701 \mathrm{~kJ} / 100 \mathrm{~g})$ were significantly higher in the most deprived households. The slope and relative indices of inequality showed that inequalities in intakes between 2001 and 2009 have changed very little.

Conclusions: There was no evidence to suggest that the difference in targeted food and nutrient intakes between the least and most deprived has decreased compared with previous years.
\end{abstract}



There is a longstanding recognition that poor dietary habits contribute to increased risk of chronic diseases such as $\mathrm{CHD}$, obesity, type 2 diabetes, hypertension, stroke and certain types of cancer, with many individuals likely to be presenting with more than one of the aforementioned diseases $^{(1)}$. Obesity, for example, has been described as the most important avoidable cause of cancer in nonsmokers in the UK, with predictions that it will eventually become the main risk factor ${ }^{(2)}$. Globally, the increase of high BMI has led to obesity becoming the leading risk factor to the burden of disease in Australasia and southern Latin America, and a high-ranking risk factor in other high-income regions in North Africa, the Middle East and
Oceania $^{(3)}$. Scotland has one of the highest obesity rates $\left(\mathrm{BMI} \geq 30 \cdot 0 \mathrm{~kg} / \mathrm{m}^{2}\right.$ ) in the world with predictions that over $40 \%$ of the population will be obese by $2030^{(4)}$.

In the UK, poor diet poses the largest economic burden to the National Health Service, with overweight and obesity being one of the main contributing factors ${ }^{(5)}$. It has been estimated that poor diet-related ill health cost the UK National Health Service $£ 5.8$ billion in 2006-2007, followed by alcohol ( $\$ 3 \cdot 3$ billion), smoking ( $\$ 3 \cdot 3$ billion) and physical inactivity (£0.9 billion) related ill health, respectively ${ }^{(5)}$. The relationship between low socioeconomic position and health has been well documented over the last three decades ${ }^{(6-9)}$, and it is well established 
that increasing deprivation is associated with a poorer $\operatorname{diet}^{(10-12)}$ and poorer associated health outcomes ${ }^{(13)}$. Following the publication of Fair Society, Healthy Lives ${ }^{(9)}$, the Marmot Review Team has worked with local authorities and primary care trusts across England to assist them to implement the recommendations set out in the review. In Scotland, the Scottish Government's ministerial task force on health inequalities, involved in the publication of recommendations in Equally Well ${ }^{(14)}$, the Early Years Framework ${ }^{(15)}$ and Achieving Our Potential ${ }^{(16)}$, re-convened in 2010 to review progress made with the implementation of the three frameworks and to highlight areas for more intense effort ${ }^{(17)}$.

Scottish Dietary Targets were set in the 1996 Scottish Diet Action Plan, Eating for Health: A Diet Action Plan for Scotland ${ }^{(18)}$, following the recognition of the need to tackle poor diet and obesity in Scotland in the 1993 Scottish Office report on the Scottish diet ${ }^{(19)}$. It has been acknowledged that progress towards these goals is slow and Lang et al. suggested that this is due to a failure to eliminate the inequalities in dietary patterns ${ }^{(20)}$.

These inequalities are linked to social deprivation, which has been reported in many ways including measures based on income ${ }^{(21)}$, equivalised income (taking into account household size and composition), education and social class ${ }^{(22,23)}$, and area-based measures such as the English and Scottish Indices of Multiple Deprivation ${ }^{(24,25)}$. The Scottish Index of Multiple Deprivation (SIMD) is based on indicators within seven individual domains of: (i) current income; (ii) employment; (iii) housing; (iv) health; (v) education, skills and training; (vi) geographic access to services and telecommunications; and (vii) crime (which was added in 2006). SIMD is presented at data zone level, each containing around 350 households, which are ranked from most deprived (1) to least deprived (6505) enabling small pockets of deprivation to be identified, and is usually summarised in quintiles or deciles.

It is useful to be able to describe inequalities in absolute and relative terms rather than simply relying on the extremes of deprivation. The Relative Index of Inequality (RII) and the Slope Index of Inequality (SII) take into account inequalities across the socio-economic scale. The SII is a measure of absolute inequality ${ }^{(26)}$, which can reflect socio-economic dimensions to inequalities in health status. The SII is the linear regression coefficient which shows the relationship between a measure of health status (or in the present paper, the mean food or nutrient intake) in each socio-economic position and the ranking of each socio-economic position. However, SII is sensitive to the mean intake of the population as absolute differences can widen but relative differences remain the same. In order to obtain relative differences, the SII can be divided by the mean measure of health status of the population to obtain the RII. In the present paper, the RII and SII are used to compare the gradients of food and nutrient intake. To our knowledge this is the first time that this has been done.
Wrieden et $a l{ }^{(27)}$ have monitored the Scottish diet at a population level using household purchase data for Scotland from the UK Living Costs and Food Survey (LCFS; formerly the Expenditure and Food Survey (EFS)) from 2001 to 2009. They have shown that there have been very slight positive changes in dietary intake between 2001 and 2009, but that targets are still not being met. However, despite these population-level changes there is little evidence to suggest that health campaigns targeted at the most deprived are reducing any inequalities in dietary intake.

The aims of this current work were to: (i) explore the association between diet and socio-economic position using household purchase data and an area-based measure of deprivation for 2007 to 2009; and (ii) investigate trends in socio-economic inequalities in diet/intake between 2001 and 2009 .

\section{Methods}

The present study used food purchase data from the Scottish sample of the UK EFS (2001-2007) and LCFS (2008-2009) $^{(28)}$ to estimate household-level consumption. A representative sample of about 550 households from Scotland was recruited to the EFS or LCFS each year. As part of the survey, individuals within each household completed a detailed $14 \mathrm{~d}$ diary of all foods and beverages purchased for consumption both in and out of the home. From these data, the mean food and nutrient consumption per person was derived. The present study examines the data from the Scottish households in the survey between 2001 and 2009. Data for each year, in raw form, were obtained from the UK Data Archive, University of Essex. Sampling variables for the EFS/LCFS households and the SIMD were obtained from the UK Office of National Statistics and Scottish Neighbourhood Statistics, respectively.

The methodology for calculating mean food and nutrient intakes from the EFS/LCFS, including details on waste and adjustment factors, is described elsewhere ${ }^{(27,29)}$. In brief, the mean daily food consumption per person was calculated from the $14 \mathrm{~d}$ diary, adjusted for waste and for the number of individuals in the household. The mean daily nutrient intake per person was calculated based on the nutrient content of the foods and adjusted for waste and the number of individuals in the household. The EFS/LCFS food composition database from the Department for Environment Food \& Rural Affairs was used to calculate nutrient intake (the data for this being supplied by the Food Standards Agency from the National Diet and Nutrition Survey (NDNS)). The SIMD for each household was added to the data file and the established methodology ${ }^{(27,29)}$ was utilised to calculate mean food and nutrient intakes by SIMD quintile. Data were analysed in 3-year groupings (i.e. 2001 to 2003; 2004 to 2006; 2007 to 2009) in order to increase sample size and power. 
SIMD 2004 was used for the analysis of data from 2001 to 2006 and SIMD 2009 was used for the analysis of data from 2007 to $2009^{(25)}$.

Food consumption and nutrient intakes (means with $95 \%$ confidence intervals) relating to the Scottish Dietary Targets, energy density and other foods, drinks and nutrients indicative of diet quality (as described by Wrieden et $a l^{(27,29)}$ ) were calculated for each of the combined 3-year data groupings from the EFS/LCFS by quintiles of the SIMD. Linear associations between food consumption/ nutrient intake and SIMD quintile were assessed by linear regression.

The SII was calculated as a measure of inequality of food consumption and nutrient intake. The SII was derived by ranking each household by SIMD (within the 3-year groupings 2001 to 2003, 2004 to 2006, and 2007 to 2009). The rank scores obtained were divided by the sample size (for the appropriate 3-year grouping) to obtain a value between 0 and 1, weighted to the relative distribution across SIMD quintiles. Linear regression analysis (weighted least squares) of the mean intake within each SIMD quintile was used to calculate the SII for each food/ nutrient. The regression (or slope) coefficient from the regression analysis is the SII. For interpretation purposes, the SII is the mean difference in intake between the hypothetically most deprived relative to the hypothetically least deprived person in the population ${ }^{(30)}$. A positive figure indicates that consumption/intake is highest in the least deprived and a negative figure indicates that consumption/intake is highest in the most deprived. In order to compare a measure of inequality across populations or years, the RII was calculated, which is the SII divided by the overall population mean food consumption or nutrient intake. The RII is the absolute difference between the most and least deprived group as a proportion of average intake in the population and thus allows comparisons across different years, when levels of intake may change. For both SII and RII, the underlying assumption is that there is a linear gradient of intake across the deprivation variable.

\section{Results}

A total of 5020 households (11374 people), over the period 2001-2009, were in the sample analysed for the present study (1537 households and 3371 people for the period 2007 to 2009). Tables 1 and 2 present results on food, energy density and nutrient intakes by SIMD quintile for the most recent 3-year period (2007 to 2009). Data for 2001 to 2003 and 2004 to 2006 are available elsewhere $^{(31)}$. Table 1 highlights that a clear gradient was found in fruit and vegetable consumption by SIMD, with consumption in the most deprived quintile (quintile 1) being $200 \mathrm{~g} / \mathrm{d}$ compared with $348 \mathrm{~g} / \mathrm{d}$ in the least deprived quintile (quintile 5) for 2007 to 2009. This positive linear trend was highly significant $(P<0 \cdot 001)$. Consumption of brown/wholemeal bread, breakfast cereals (all types and wholegrain/high fibre), oil-rich fish and white fish were highest in the least deprived quintile. Total bread consumption was highest in the most deprived quintile and there was no difference in fresh potato consumption by deprivation quintiles for this period. With regard to nutrient targets, no linear associations were found in the percentage of food energy from total fat and saturated fat and in the intake of complex carbohydrates (starch and NSP) by SIMD quintile for 2007 to 2009 (Table 1). Non-milk extrinsic sugars (NMES) intake was significantly higher in the most deprived quintile at $15.5 \%$ of food energy, compared with $14.3 \%$ of food energy in the least deprived quintile, which also had the highest consumption of sugar-containing soft drinks (Table 2). A significant linear association was also found in intake of NSP by SIMD quintile with intakes significantly lower in the most deprived quintile at $11.4 \mathrm{~g} / \mathrm{d}$, compared with $13.8 \mathrm{~g} / \mathrm{d}$ in the least deprived quintile. Energy density was significantly higher in the most deprived quintile $(741 \mathrm{~kJ} /$ $100 \mathrm{~g})$ compared with the least deprived quintile $(701 \mathrm{~kJ} /$ $100 \mathrm{~g}$ ). By 2009, with the exception of mean consumption of white fish in the least deprived quintile of SIMD, none of the Scottish Dietary Targets were achieved on average within any of the SIMD quintiles.

Results for foods contributing NMES are given in Table 2. As previously mentioned, sugar-containing soft drink consumption was significantly higher in the most deprived quintile of SIMD with mean daily consumption of $284 \mathrm{~g}$ compared with $180 \mathrm{~g}$ in the least deprived quintile, which equates to a difference of approximately one-third of a standard can per day (Table 2). Conversely, consumption of cakes and pastries was lowest in the most deprived quintile, with intakes of $15 \mathrm{~g} / \mathrm{d}$ compared with $20 \mathrm{~g} / \mathrm{d}$ in the least deprived quintile. Consumption of foods in the other red meat products group (includes the meat portion of meat pies, sausages, corned beef, burgers and pâté), whole milk, processed potatoes and takeaway foods were significantly higher in the most deprived quintile of SIMD (Table 2). Mean consumption of whole milk was more than double for the most deprived compared with the least deprived quintile.

SII and RII values with 95\% CI were calculated for 2001-2003, 2004-2006 and 2007-2009 to allow for a comparison to be made over time of absolute and relative differences. While they were calculated for all foods and nutrients, it is acknowledged that a linear difference was not found for all foods and nutrients for each of the 3-year time periods. Tables 3 and 4 show that absolute and relative inequalities in food/nutrient intakes have not changed appreciably between 2001 and 2009. The magnitude of the inequalities is substantial for some foods, e.g. fruit and vegetables where the mean intake in the most deprived was the equivalent of two portions less than in the least deprived. A significant difference was found for SII for sugar-free soft drink consumption; however, this is 
Table 1 Consumption of foods and nutrients targeted in the Scottish Diet Action Plan 1996 ${ }^{(18)}$, by SIMD quintile, 2007 to 2009 , combined EFS/LCFS data (g/person per d for foods with the exception of fish, g/person per week; units/person per $d$ for nutrients)

\begin{tabular}{|c|c|c|c|c|c|c|c|c|c|c|c|c|}
\hline \multirow[b]{2}{*}{ Food/nutrient } & \multirow[b]{2}{*}{ Scottish Dietary Target } & \multicolumn{2}{|c|}{$\begin{array}{l}\text { SIMD quintile } 1 \\
\text { most deprived }\end{array}$} & \multicolumn{2}{|c|}{ SIMD quintile 2} & \multicolumn{2}{|c|}{ SIMD quintile 3} & \multicolumn{2}{|c|}{ SIMD quintile 4} & \multicolumn{2}{|c|}{$\begin{array}{l}\text { SIMD quintile } 5 \\
\text { least deprived }\end{array}$} & \multirow{2}{*}{$\begin{array}{l}P \text { value for linear } \\
\text { association }\end{array}$} \\
\hline & & Mean & $95 \% \mathrm{Cl}$ & Mean & $95 \% \mathrm{Cl}$ & Mean & $95 \% \mathrm{Cl}$ & Mean & $95 \% \mathrm{Cl}$ & Mean & $95 \% \mathrm{Cl}$ & \\
\hline \multicolumn{13}{|l|}{ Foods } \\
\hline Fruit and vegetables $(\mathrm{g})^{\star}, \dagger$ & $400 \mathrm{~g} / \mathrm{d}$ & 200 & 177,223 & 260 & 231,288 & 284 & 261,306 & 298 & 264,332 & 348 & 326,369 & $<0.001$ \\
\hline Fruit $(\mathrm{g})^{*}$ & & 103 & $86 \cdot 3,120$ & 133 & 116,149 & 154 & 134,174 & 159 & 133,185 & 200 & 186,215 & $<0.001$ \\
\hline Vegetables $(\mathrm{g}) \dagger$ & & $97 \cdot 0$ & $85 \cdot 3,109$ & 127 & 112,142 & 129 & 115,144 & 139 & 125,153 & 148 & 137,159 & $<0.001$ \\
\hline Total bread $(\mathrm{g})$ & $154 \mathrm{~g} / \mathrm{d}$ & 105 & $96 \cdot 9,113$ & 97.5 & $90 \cdot 6,104$ & 91.1 & $85 \cdot 6,96 \cdot 6$ & 93.7 & $87 \cdot 4,100$ & 91.5 & $87.0,95.9$ & 0.004 \\
\hline Brown/wholemeal bread (g) & & $17 \cdot 2$ & $14 \cdot 3,20 \cdot 2$ & $23 \cdot 0$ & $19 \cdot 3,26 \cdot 8$ & $22 \cdot 3$ & $18 \cdot 7,25 \cdot 8$ & $23 \cdot 1$ & $19 \cdot 8,26 \cdot 3$ & $26 \cdot 5$ & $24 \cdot 0,29 \cdot 0$ & 0.001 \\
\hline Total breakfast cereal $(\mathrm{g})$ & $34 \mathrm{~g} / \mathrm{d}$ & $15 \cdot 7$ & $12 \cdot 3,19 \cdot 0$ & 19.7 & $17 \cdot 4,22 \cdot 1$ & $22 \cdot 7$ & $20 \cdot 2,25 \cdot 3$ & $24 \cdot 3$ & $20 \cdot 0,28 \cdot 6$ & $27 \cdot 0$ & $24 \cdot 4,29 \cdot 6$ & $<0.001$ \\
\hline High-fibre breakfast cereal (g) & & 7.5 & $5 \cdot 2,9 \cdot 8$ & 10.9 & $9 \cdot 3,12 \cdot 6$ & 14.0 & $11 \cdot 9,16 \cdot 2$ & $15 \cdot 1$ & $11.9,18.4$ & $17 \cdot 2$ & $15 \cdot 3,19 \cdot 1$ & $<0.001$ \\
\hline Oil-rich fish (g) & $88 \mathrm{~g} /$ week & $20 \cdot 8$ & $13 \cdot 0,28 \cdot 6$ & $25 \cdot 2$ & $21 \cdot 8,28 \cdot 6$ & $32 \cdot 0$ & $24.4,39.5$ & 37.9 & $28 \cdot 4,47.5$ & 39.9 & $31 \cdot 2,48 \cdot 7$ & $<0.001$ \\
\hline White fish (g) & No decreaseף & $77 \cdot 2$ & $60 \cdot 4,94 \cdot 0$ & 90.7 & $77 \cdot 5,104$ & $87 \cdot 7$ & $73 \cdot 3,102$ & $97 \cdot 3$ & $81 \cdot 3,113$ & 112 & $95 \cdot 8,128$ & 0.008 \\
\hline Fresh potatoes $(\mathrm{g}) \ddagger$ & & 48.9 & $40 \cdot 0,57 \cdot 7$ & 51.3 & $44.8,57.9$ & $49 \cdot 8$ & $41 \cdot 5,58 \cdot 0$ & $61 \cdot 2$ & $49 \cdot 3,73 \cdot 1$ & $51 \cdot 0$ & $47 \cdot 0,55 \cdot 1$ & 0.283 \\
\hline \multicolumn{13}{|l|}{ Energy and nutrients } \\
\hline Fat ( $\%$ of food energy) & $\leq 35 \%$ & 38.8 & $37 \cdot 6,39 \cdot 9$ & 39.1 & $38 \cdot 2,40 \cdot 0$ & 38.6 & $37.8,39.5$ & 39.1 & $38 \cdot 1,40 \cdot 2$ & 38.7 & $38 \cdot 1,39 \cdot 3$ & 0.922 \\
\hline Saturated fat (\% of food energy) & $\leq 11 \%$ & $15 \cdot 2$ & $14 \cdot 8,15 \cdot 7$ & $15 \cdot 0$ & $14 \cdot 6,15 \cdot 3$ & $15 \cdot 3$ & $14 \cdot 8,15 \cdot 8$ & $15 \cdot 3$ & $14 \cdot 9,15 \cdot 8$ & $15 \cdot 4$ & $15 \cdot 0,15 \cdot 7$ & 0.281 \\
\hline NMES (\% of food energy) & $\begin{array}{l}\text { Adults: No } \uparrow^{* *} \\
\text { Children: }<10 \%\end{array}$ & $15 \cdot 5$ & $14 \cdot 6,16 \cdot 5$ & 14.9 & $14 \cdot 0,15 \cdot 8$ & $15 \cdot 6$ & $14.4,16 \cdot 8$ & 14.5 & $13 \cdot 9,15 \cdot 1$ & $14 \cdot 3$ & $13 \cdot 7,14 \cdot 8$ & 0.011 \\
\hline Complex carbohydrates (g) & $155 \mathrm{~g} / \mathrm{d}$ & 143 & 133,154 & 143 & 136,150 & 144 & 136,152 & 147 & 136,157 & 147 & 142,153 & 0.346 \\
\hline NSP $(g) \S$ & & 11.4 & $10 \cdot 5,12 \cdot 3$ & 12.4 & $11 \cdot 7,13 \cdot 2$ & 12.6 & $11.9,13.3$ & 13.1 & $12 \cdot 0,14 \cdot 2$ & 13.8 & $13 \cdot 2,14 \cdot 4$ & $<0.001$ \\
\hline Food energy (kJ) & & 8496 & 7937, 9054 & 8492 & 8049,8934 & 8560 & 8168,8953 & 8642 & 8136,9148 & 8607 & 8283,8931 & 0.625 \\
\hline Energy density $(\mathrm{kJ} / 100 \mathrm{~g})$ & & 741 & 719,764 & 725 & 705,744 & 717 & 700,734 & 731 & 705,756 & 701 & 683,719 & 0.025 \\
\hline No. of households & & & 270 & & 309 & & 303 & & 301 & & 354 & \\
\hline No. of people & & & 540 & & 658 & & 656 & & 665 & & 852 & \\
\hline No. of people, weightedll & & & 2495 & & 2974 & & 2948 & & 3057 & & 3882 & \\
\hline
\end{tabular}

SIMD, Scottish Index of Multiple Deprivation; EFS, Expenditure and Food Survey; LCFS, Living Costs and Food Survey; NMES, non-milk extrinsic sugars.

Household and eating out consumption combined. Significant $P$ values are indicated in bold font.

*Fruit includes fruit and vegetable juice.

†Vegetables include baked beans.

†Part of complex carbohydrate target.

Sietary Reference Value $=18 \mathrm{~g}$

The results are weighted to the Scottish population; the number provided is approximately 1000th of the Scottish population.

TValue from the National Food Survey for 1996 reported by Wrieden et al. (2006) ${ }^{(54)}$ was $107 \mathrm{~g} / \mathrm{week}$.

${ }^{* *}$ Dietary Reference Value for adults $=11 \%$ of food energy ${ }^{(53)}$. 
Table 2 Consumption of additional foods and drinks indicative of diet quality by SIMD quintile, 2007 to 2009, combined EFS/LCFS data (g/person per d)

\begin{tabular}{|c|c|c|c|c|c|c|c|c|c|c|c|}
\hline \multirow[b]{2}{*}{ Food } & \multicolumn{2}{|c|}{$\begin{array}{l}\text { SIMD quintile } 1 \\
\text { most deprived }\end{array}$} & \multicolumn{2}{|c|}{ SIMD quintile 2} & \multicolumn{2}{|c|}{ SIMD quintile 3} & \multicolumn{2}{|c|}{ SIMD quintile 4} & \multicolumn{2}{|c|}{$\begin{array}{l}\text { SIMD quintile } 5 \\
\text { least deprived }\end{array}$} & \multirow{2}{*}{$\begin{array}{c}P \text { value for linear } \\
\text { association }\end{array}$} \\
\hline & Mean & $95 \% \mathrm{Cl}$ & Mean & $95 \% \mathrm{Cl}$ & Mean & $95 \% \mathrm{Cl}$ & Mean & $95 \% \mathrm{Cl}$ & Mean & $95 \% \mathrm{Cl}$ & \\
\hline Cakes and pastries & $15 \cdot 2$ & $12 \cdot 8,17 \cdot 6$ & $15 \cdot 2$ & $13 \cdot 3,17 \cdot 1$ & $18 \cdot 0$ & $15 \cdot 8,20 \cdot 2$ & $18 \cdot 4$ & $15 \cdot 4,21 \cdot 3$ & $19 \cdot 7$ & $17 \cdot 4,22 \cdot 0$ & 0.006 \\
\hline Sweet biscuits & $24 \cdot 2$ & $20 \cdot 5,28 \cdot 0$ & $21 \cdot 2$ & $18 \cdot 6,23 \cdot 8$ & $25 \cdot 5$ & $21 \cdot 1,29 \cdot 9$ & $23 \cdot 2$ & $19 \cdot 3,27 \cdot 1$ & $24 \cdot 0$ & $21 \cdot 7,26 \cdot 3$ & 0.692 \\
\hline Cakes, sweet biscuits and pastries & 39.4 & $34.0,44.9$ & $36 \cdot 4$ & $32 \cdot 7,40 \cdot 1$ & 43.5 & $38.0,48.9$ & 41.5 & $35 \cdot 2,47 \cdot 8$ & 43.7 & $40 \cdot 3,47 \cdot 0$ & 0.070 \\
\hline Sugar and preserves & $16 \cdot 4$ & $13 \cdot 4,19 \cdot 3$ & 17.5 & $12 \cdot 0,23 \cdot 0$ & 18.4 & $16 \cdot 0,20 \cdot 9$ & $22 \cdot 1$ & $17 \cdot 9,26 \cdot 2$ & $15 \cdot 8$ & $13 \cdot 3,18 \cdot 2$ & 0.779 \\
\hline Chocolate confectionery & $13 \cdot 6$ & $11 \cdot 4,15 \cdot 7$ & $15 \cdot 5$ & $11 \cdot 9,19 \cdot 2$ & $16 \cdot 6$ & $14 \cdot 1,19 \cdot 1$ & $14 \cdot 1$ & $11 \cdot 4,16 \cdot 8$ & $16 \cdot 1$ & $13 \cdot 8,18 \cdot 4$ & 0.443 \\
\hline Sugar confectionery & 7.9 & $6 \cdot 1,9 \cdot 8$ & 6.8 & $5 \cdot 8,7 \cdot 8$ & 6.7 & $5 \cdot 5,8.0$ & $6 \cdot 7$ & $5 \cdot 2,8 \cdot 2$ & $6 \cdot 1$ & $4 \cdot 8,7 \cdot 4$ & 0.151 \\
\hline Total confectionery & 21.5 & $18 \cdot 1,25 \cdot 0$ & $22 \cdot 3$ & $18 \cdot 4,26 \cdot 3$ & 23.3 & $20 \cdot 3,26 \cdot 3$ & $20 \cdot 7$ & $17 \cdot 5,24 \cdot 0$ & $22 \cdot 2$ & $19.5,24.9$ & 0.958 \\
\hline Sugar-containing soft drinks & 284 & 244,325 & 246 & 207,286 & 214 & 186, 242 & 175 & 155,195 & 180 & 157, 203 & $<0.001$ \\
\hline Sugar-free soft drinks & $93 \cdot 0$ & $66 \cdot 3,120$ & 93.7 & $70 \cdot 4,117$ & 76.9 & $62 \cdot 1,91 \cdot 8$ & $97 \cdot 2$ & $73 \cdot 5,121$ & 82.4 & $63 \cdot 8,101$ & 0.629 \\
\hline Total soft drinks & 377 & 320,434 & 340 & 295,385 & 291 & 256,326 & 272 & 237,308 & 262 & 229,295 & $<0.001$ \\
\hline Total red meat ${ }^{*}$ & $63 \cdot 7$ & $56 \cdot 8,70 \cdot 6$ & 64.9 & $58.9,70.9$ & $60 \cdot 3$ & $55 \cdot 4,65 \cdot 2$ & $62 \cdot 4$ & $53 \cdot 5,71 \cdot 3$ & $57 \cdot 0$ & $52 \cdot 0,62 \cdot 0$ & 0.117 \\
\hline Bacon and ham & $10 \cdot 7$ & $8 \cdot 8,12 \cdot 5$ & $12 \cdot 5$ & $10 \cdot 9,14.2$ & $12 \cdot 7$ & $11 \cdot 7,13.8$ & $12 \cdot 3$ & $10 \cdot 5,14 \cdot 1$ & $12 \cdot 5$ & $11 \cdot 1,14.0$ & 0.275 \\
\hline Other red meat products ${ }^{*}, \dagger$ & 33.4 & $29.2,37.5$ & $30 \cdot 6$ & $27 \cdot 5,33 \cdot 8$ & $25 \cdot 8$ & $22 \cdot 7,28 \cdot 8$ & $24 \cdot 6$ & $22 \cdot 0,27 \cdot 3$ & 23.0 & $20 \cdot 0,25 \cdot 9$ & $<0.001$ \\
\hline Butter & 5.9 & $4 \cdot 7,7 \cdot 2$ & $5 \cdot 1$ & $3 \cdot 9,6 \cdot 2$ & $7 \cdot 6$ & $6 \cdot 0,9 \cdot 1$ & $7 \cdot 0$ & $5 \cdot 6,8 \cdot 3$ & $6 \cdot 7$ & $5 \cdot 3,8 \cdot 0$ & 0.138 \\
\hline Whole milk & $91 \cdot 1$ & $65 \cdot 4,117$ & 59.4 & $47 \cdot 0,71 \cdot 7$ & $60 \cdot 4$ & $44.4,76 \cdot 4$ & 41.9 & $30 \cdot 4,53 \cdot 3$ & $43 \cdot 2$ & $28 \cdot 3,58 \cdot 1$ & 0.001 \\
\hline Semi-skimmed milk & 135 & 106,164 & 128 & 108,148 & 142 & 124,160 & 146 & 122,170 & 138 & 119,157 & 0.521 \\
\hline Skimmed milk & $11 \cdot 7$ & $4.9,18.5$ & $13 \cdot 3$ & $8 \cdot 2,18.5$ & $22 \cdot 9$ & $14 \cdot 5,31 \cdot 2$ & $17 \cdot 3$ & $10 \cdot 3,24 \cdot 4$ & $17 \cdot 9$ & $12 \cdot 2,23 \cdot 5$ & 0.129 \\
\hline Total milk & 252 & 227,278 & 223 & 205,241 & 237 & 217,258 & 223 & 201,245 & 224 & 206, 242 & 0.101 \\
\hline Processed potatoes & 35.4 & $30 \cdot 6,40 \cdot 2$ & $32 \cdot 2$ & $28 \cdot 1,36 \cdot 2$ & 27.6 & $24 \cdot 2,31 \cdot 0$ & $25 \cdot 7$ & $21 \cdot 8,29 \cdot 7$ & 22.9 & $19 \cdot 6,26 \cdot 2$ & $<0.001$ \\
\hline Savoury snacks & $14 \cdot 0$ & $12 \cdot 2,15 \cdot 8$ & $13 \cdot 3$ & $11 \cdot 7,15 \cdot 0$ & $13 \cdot 4$ & $11 \cdot 7,15 \cdot 1$ & $12 \cdot 8$ & $10 \cdot 7,14.9$ & $12 \cdot 3$ & $10 \cdot 3,14 \cdot 4$ & 0.182 \\
\hline Takeaway foods & $24 \cdot 2$ & $19 \cdot 1,29 \cdot 4$ & 24.8 & $20 \cdot 4,29 \cdot 3$ & $17 \cdot 7$ & $14 \cdot 4,21 \cdot 1$ & $16 \cdot 7$ & $13 \cdot 5,19 \cdot 9$ & $18 \cdot 3$ & $14 \cdot 8,21.9$ & 0.008 \\
\hline No. of households & & \multicolumn{2}{|c|}{309} & \multicolumn{2}{|c|}{303} & \multicolumn{2}{|r|}{301} & \multicolumn{2}{|c|}{354} & \\
\hline No. of people & \multirow{2}{*}{\multicolumn{2}{|c|}{$\begin{array}{r}540 \\
495\end{array}$}} & \multicolumn{2}{|c|}{$\begin{array}{r}658 \\
2974\end{array}$} & \multirow{2}{*}{\multicolumn{2}{|c|}{$\begin{array}{r}656 \\
2948\end{array}$}} & \multirow{2}{*}{\multicolumn{2}{|c|}{$\begin{array}{r}665 \\
3057\end{array}$}} & \multirow{2}{*}{\multicolumn{2}{|c|}{$\begin{array}{r}852 \\
3882\end{array}$}} & \\
\hline No. of people, weighted & & & \multicolumn{2}{|c|}{2974} & & & & & & & \\
\hline
\end{tabular}

SIMD, Scottish Index of Multiple Deprivation; EFS, Expenditure and Food Survey; LCFS, Living Costs and Food Survey.

Household and eating out intakes combined. Significant $P$ values are indicated in bold font.

${ }^{*}$ Meat portion only; see appendices $2 \& 4$ of Barton et al. (2012) ${ }^{(31)}$ for methodology.

tOther red meat products include the meat portion of sausages, meat pies, corned beef, burgers and pâté, and is a component of total red meat.

FThe results are weighted to the Scottish population; the number provided is approximately 1000th of the Scottish population. 
Table 3 SII and RII for the relationship of SIMD quintiles on foods and nutrients targeted in the Scottish Diet Action Plan 1996 ${ }^{(18)}$, EFS/LCFS data

\begin{tabular}{|c|c|c|c|c|c|c|c|c|c|c|c|c|c|c|}
\hline \multirow[b]{2}{*}{ Food/nutrient } & \multirow{2}{*}{$\begin{array}{l}\text { Scottish } \\
\text { Dietary Target }\end{array}$} & \multicolumn{2}{|c|}{ 2001-2003 (SII)* } & \multicolumn{2}{|c|}{ 2004-2006† (SII)* } & \multicolumn{2}{|c|}{ 2007-2009 (SII)* } & \multirow{2}{*}{$\begin{array}{l}P \text { value for linear } \\
\text { association for SII }\end{array}$} & \multicolumn{2}{|c|}{ 2001-2003 (RII) } & \multicolumn{2}{|c|}{ 2004-2006† (RII) } & \multicolumn{2}{|c|}{ 2007-2009 (RII) } \\
\hline & & Mean & $95 \% \mathrm{Cl}$ & Mean & $95 \% \mathrm{Cl}$ & Mean & $95 \% \mathrm{Cl}$ & & Mean & $95 \% \mathrm{Cl}$ & Mean & $95 \% \mathrm{Cl}$ & Mean & $95 \% \mathrm{Cl}$ \\
\hline \multicolumn{15}{|l|}{ Foods } \\
\hline $\begin{array}{l}\text { Fruit and vegetables } \\
(\mathrm{g}) \ddagger, \S\end{array}$ & $400 \mathrm{~g} / \mathrm{d}$ & 167 & 132,202 & 157 & 124,190 & 166 & 128,204 & 0.905 & 0.65 & $0.51,0.79$ & 0.57 & $0.45,0.69$ & 0.58 & $0.45,0.72$ \\
\hline Fruit $(\mathrm{g}) \ddagger$ & & 119 & $95 \cdot 5,143$ & 104 & $81 \cdot 9,126$ & 112 & $85 \cdot 3,138$ & 0.664 & 0.90 & $0.72,1.07$ & 0.71 & $0.56,0.86$ & 0.72 & $0.55,0.90$ \\
\hline Vegetables $(\mathrm{g}) \S$ & & $47 \cdot 7$ & $31.4,63.9$ & $52 \cdot 9$ & $34 \cdot 1,71 \cdot 8$ & $54 \cdot 3$ & $35 \cdot 2,73 \cdot 3$ & 0.852 & 0.39 & $0.25,0.52$ & 0.41 & $0.27,0.56$ & 0.42 & $0.27,0.56$ \\
\hline Total bread $(\mathrm{g})$ & $154 \mathrm{~g} / \mathrm{d}$ & $-17 \cdot 7$ & $-28 \cdot 7,-6 \cdot 6$ & $-10 \cdot 8$ & $-22 \cdot 6,1.0$ & $-14 \cdot 1$ & $-23 \cdot 5,-4 \cdot 7$ & 0.715 & -0.16 & $-0.27,-0.06$ & -0.11 & $-0.22,0.01$ & -0.15 & $-0.25,-0.05$ \\
\hline $\begin{array}{l}\text { Brown/wholemeal bread } \\
\text { (g) }\end{array}$ & & $12 \cdot 6$ & $8 \cdot 2,17 \cdot 1$ & $9 \cdot 6$ & $4 \cdot 4,14 \cdot 8$ & $9 \cdot 1$ & $4 \cdot 0,14 \cdot 2$ & 0.542 & $0 \cdot 70$ & $0.46,0.95$ & 0.42 & $0.19,0.65$ & 0.40 & $0.18,0.62$ \\
\hline Total breakfast cereal (g) & $34 \mathrm{~g} / \mathrm{d}$ & $10 \cdot 9$ & $6 \cdot 6,15 \cdot 3$ & $13 \cdot 8$ & $9 \cdot 7,17 \cdot 9$ & 13.4 & $8 \cdot 3,18 \cdot 4$ & 0.604 & 0.56 & $0.34,0.78$ & 0.70 & $0.49,0.90$ & 0.60 & $0.37,0.82$ \\
\hline $\begin{array}{l}\text { High-fibre breakfast cereal } \\
\text { (g) }\end{array}$ & & 9.5 & $5 \cdot 9,13 \cdot 0$ & $10 \cdot 5$ & $7 \cdot 1,13 \cdot 8$ & 11.5 & $8 \cdot 3,14 \cdot 8$ & 0.691 & 0.92 & $0.57,1.26$ & 0.95 & $0.64,1.24$ & 0.86 & $0.62,1.10$ \\
\hline Oil-rich fish (g) & $88 \mathrm{~g} /$ week & $25 \cdot 4$ & $12 \cdot 8,38 \cdot 1$ & 34.2 & $19 \cdot 0,49 \cdot 4$ & $25 \cdot 1$ & $13 \cdot 7,36 \cdot 5$ & 0.589 & 0.81 & $0.41,1.22$ & 0.89 & $0.49,1.28$ & 0.78 & $0.43,1.14$ \\
\hline White fish $(\mathrm{g})$ & No decrease†† & $26 \cdot 1$ & $6 \cdot 3,46 \cdot 0$ & 36.5 & $19 \cdot 0,54 \cdot 0$ & $39 \cdot 1$ & $10 \cdot 9,67 \cdot 3$ & 0.692 & 0.28 & $0.07,0.49$ & 0.41 & $0.21,0.60$ & 0.41 & $0.12,0.71$ \\
\hline Fresh potatoes $(\mathrm{g}) \|$ & & -10.9 & $-20 \cdot 7,-1 \cdot 0$ & 2.8 & $-9.5,15.1$ & $5 \cdot 8$ & $-5 \cdot 3,16 \cdot 8$ & 0.06 & -0.18 & $-0.35,-0.02$ & 0.05 & $-0.17,0.27$ & 0.11 & $-0.10,0.32$ \\
\hline \multicolumn{15}{|l|}{ Energy and nutrients } \\
\hline Fat (\% of food energy) & $\leq 35 \%$ & -0.2 & $-1.4,1 \cdot 1$ & -0.6 & $-1 \cdot 8,0.5$ & -0.1 & $-1 \cdot 7,1 \cdot 6$ & 0.832 & -0.01 & $-0.04,0.03$ & -0.02 & $-0.05,0.01$ & 0.00 & $-0.04,0.04$ \\
\hline $\begin{array}{l}\text { Saturated fat (\% of food } \\
\text { energy) }\end{array}$ & $\leq 11 \%$ & 0.1 & $-0.4,0.6$ & 0.2 & $-0.5,0.9$ & 0.3 & $-0.3,0.9$ & 0.848 & 0.01 & $-0.03,0.04$ & 0.01 & $-0.03,0.06$ & 0.02 & $-0.02,0.06$ \\
\hline NMES ( $\%$ of food energy) & $\begin{array}{c}\text { Adults: } \\
\text { No } \uparrow \neq \ddagger \\
\text { Children: }<10 \%\end{array}$ & $-2 \cdot 4$ & $-3 \cdot 7,-1 \cdot 1$ & $-2 \cdot 2$ & $-3 \cdot 8,-0.6$ & $-1 \cdot 5$ & $-2 \cdot 6,-0 \cdot 4$ & 0.527 & -0.15 & $-0.24,-0.07$ & -0.14 & $-0.25,-0.04$ & -0.10 & $-0.17,-0.03$ \\
\hline $\begin{array}{l}\text { Complex carbohydrates } \\
\text { (g) }\end{array}$ & $155 \mathrm{~g} / \mathrm{d}$ & 3.6 & $-7.5,14 \cdot 8$ & $8 \cdot 2$ & $-3 \cdot 3,19 \cdot 7$ & $6 \cdot 1$ & $-6 \cdot 8,19 \cdot 1$ & 0.822 & 0.02 & $-0.05,0.10$ & 0.06 & $-0.02,0.14$ & 0.04 & $-0.05,0.13$ \\
\hline NSP $(\mathrm{g}) \boldsymbol{\Phi}$ & & $2 \cdot 4$ & $1.3,3.5$ & $2 \cdot 6$ & $1 \cdot 6,3 \cdot 6$ & $2 \cdot 7$ & $1.5,3.9$ & 0.924 & 0.20 & $0.11,0.28$ & 0.21 & $0.13,0.29$ & 0.21 & $0.12,0.30$ \\
\hline Food energy (kJ) & & $-29 \cdot 2$ & $-683,625$ & 112 & $-500,724$ & 181 & $-567,929$ & 0.901 & 0.00 & $-0.08,0.07$ & 0.01 & $-0.06,0.08$ & 0.02 & $-0.07,0.10$ \\
\hline $\begin{array}{l}\text { Energy density } \\
(\mathrm{kJ} / 100 \mathrm{~g})\end{array}$ & & $-47 \cdot 8$ & $-72 \cdot 1,-23 \cdot 4$ & $-66 \cdot 9$ & $-94.5,-39 \cdot 4$ & -38.1 & $-70 \cdot 7,-5 \cdot 5$ & 0.367 & -0.07 & $-0.10,-0.03$ & -0.09 & $-0.13,-0.06$ & -0.05 & $-0.10,-0.01$ \\
\hline No. of households & & & 1750 & & 1731 & & 1537 & & & 1750 & & 1731 & & 1537 \\
\hline No. of people & & & 4022 & & 3975 & & 3371 & & & 4022 & & 3975 & & 3371 \\
\hline No. of people, weighted ${ }^{* *}$ & & & 14935 & & 14776 & & 15356 & & & 14935 & & 14776 & & 15356 \\
\hline
\end{tabular}

SII, Slope Index of Inequality; RII, Relative Index of Inequality; SIMD, Scottish Index of Multiple Deprivation; EFS, Expenditure and Food Survey; LCFS, Living Costs and Food Survey; NMES, non-milk extrinsic sugars. Household and eating out consumption combined.

${ }^{*}$ Mean difference in intake ( $\mathrm{g} /$ person per $\mathrm{d}$ for foods with the exception of fish, $\mathrm{g} /$ person per week; units/person per $\mathrm{d}$ for nutrients) in the most deprived relative to the least deprived (slope of the gradient between the most deprived and the least deprived).

From 2006 the EFS moved from a financial year to a calendar year basis. As a consequence of this the January to March 2006 data are duplicated in the $2005 / 2006$ and the 2006 results.

\#Fruit includes fruit and vegetable juice.

§Vegetables include baked beans.

Part of complex carbohydrate target.

TDietary Reference Value $=18 \mathrm{~g}^{(5)}$

"The results are weighted to the Scottish population; the number provided is approximately 1000th of the Scottish population

††Value from the National Food Survey for 1996 reported by Wrieden et al. $(2006)^{(54)}$ was $107 \mathrm{~g} /$ week.

\#Dietary Reference Value for adults $=11 \%$ of food energy ${ }^{(53)}$ 
Table 4 SII and RII for the relationship of SIMD quintiles on additional foods and drinks indicative of diet quality, EFS/LCFS data (g/person per d)

\begin{tabular}{|c|c|c|c|c|c|c|c|c|c|c|c|c|c|}
\hline \multirow[b]{2}{*}{ Food } & \multicolumn{2}{|c|}{ 2001-2003 (SII)* } & \multicolumn{2}{|c|}{ 2004-2006† (SII)* } & \multicolumn{2}{|c|}{ 2007-2009 (SII)* } & \multirow[b]{2}{*}{$\begin{array}{l}P \text { value for linear } \\
\text { association for SII }\end{array}$} & \multicolumn{2}{|c|}{ 2001-2003 (RII) } & \multicolumn{2}{|c|}{ 2004-2006† (RII) } & \multicolumn{2}{|c|}{ 2007-2009 (RII) } \\
\hline & Mean & $95 \% \mathrm{Cl}$ & Mean & $95 \% \mathrm{Cl}$ & Mean & $95 \% \mathrm{Cl}$ & & Mean & $95 \% \mathrm{Cl}$ & Mean & $95 \% \mathrm{Cl}$ & Mean & $95 \% \mathrm{Cl}$ \\
\hline Cakes and pastries & $2 \cdot 6$ & $-1 \cdot 6,6 \cdot 9$ & 1.9 & $-2 \cdot 3,6 \cdot 0$ & $6 \cdot 1$ & $1.9,10 \cdot 3$ & 0.281 & 0.15 & $-0.09,0.40$ & 0.11 & $-0.13,0.34$ & 0.35 & $0.11,0.59$ \\
\hline Sweet biscuits & 2.7 & $-1.6,7.0$ & -0.9 & $-5.5,3.7$ & 1.0 & $-3 \cdot 8,5 \cdot 7$ & 0.511 & 0.12 & $-0.07,0.31$ & -0.04 & $-0.26,0.18$ & 0.04 & $-0.16,0.24$ \\
\hline Cakes, sweet biscuits and pastries & $5 \cdot 4$ & $-1.6,12.3$ & 1.0 & $-6 \cdot 2,8 \cdot 2$ & $7 \cdot 1$ & $-0.5,14.6$ & 0.498 & 0.14 & $-0.04,0.31$ & 0.03 & $-0.16,0.21$ & 0.17 & $-0.01,0.36$ \\
\hline Sugar and preserves & $-4 \cdot 8$ & $-9 \cdot 7,0 \cdot 1$ & $-3 \cdot 4$ & $-10 \cdot 2,3 \cdot 4$ & 0.6 & $-5 \cdot 0,6 \cdot 3$ & 0.344 & -0.26 & $-0.52,0.01$ & -0.20 & $-0.60,0.20$ & 0.03 & $-0.28,0.35$ \\
\hline Chocolate confectionery & 1.7 & $-2 \cdot 3,5 \cdot 7$ & 0.8 & $-2 \cdot 8,4 \cdot 4$ & 1.7 & $-2 \cdot 7,6 \cdot 0$ & 0.919 & 0.11 & $-0.16,0.39$ & 0.06 & $-0.20,0.31$ & 0.11 & $-0.18,0.39$ \\
\hline Sugar confectionery & -1.0 & $-2 \cdot 9,0.9$ & $-1 \cdot 1$ & $-3 \cdot 3,1 \cdot 1$ & $-1 \cdot 8$ & $-4 \cdot 3,0.7$ & 0.871 & -0.13 & $-0.37,0.12$ & -0.16 & $-0.49,0.16$ & -0.26 & $-0.63,0.10$ \\
\hline Total confectionery & 0.7 & $-4 \cdot 5,5 \cdot 8$ & -0.3 & $-5 \cdot 5,4 \cdot 8$ & $-0 \cdot 1$ & $-5 \cdot 6,5 \cdot 3$ & 0.952 & 0.03 & $-0.20,0.26$ & -0.01 & $-0.26,0.23$ & 0.00 & $-0.25,0.24$ \\
\hline Sugar-containing soft drinks & -123 & $-178,-68 \cdot 7$ & -124 & $-186,-62 \cdot 5$ & -134 & $-185,-82 \cdot 7$ & 0.954 & -0.50 & $-0.73,-0.28$ & -0.53 & $-0.79,-0.27$ & -0.62 & $-0.86,-0.38$ \\
\hline Sugar-free soft drinks & $35 \cdot 6$ & $6 \cdot 2,65 \cdot 1$ & $-40 \cdot 1$ & $-73 \cdot 4,-6 \cdot 7$ & $-9 \cdot 4$ & $-47 \cdot 8,29 \cdot 0$ & 0.007 & 0.34 & $0.06,0.63$ & -0.43 & $-0.78,-0.07$ & -0.11 & $-0.54,0.33$ \\
\hline Total soft drinks & -87.8 & $-146,-29.4$ & -164 & $-238,-90 \cdot 1$ & -143 & $-210,-76 \cdot 7$ & 0.236 & -0.25 & $-0.42,-0.08$ & -0.50 & $-0.73,-0.28$ & -0.47 & $-0.69,-0.25$ \\
\hline Total red meat & $-12 \cdot 2$ & $-19 \cdot 5,-4 \cdot 9$ & -13.9 & $-22 \cdot 8,-5 \cdot 1$ & -8.6 & $-19 \cdot 3,2 \cdot 1$ & 0.743 & -0.19 & $-0.30,-0.08$ & -0.23 & $-0.37,-0.08$ & -0.14 & $-0.31,0.03$ \\
\hline Bacon and ham & -0.6 & $-3 \cdot 3,2 \cdot 0$ & 0.3 & $-2 \cdot 6,3 \cdot 3$ & 1.5 & $-1 \cdot 3,4 \cdot 2$ & 0.547 & -0.05 & $-0.27,0.16$ & 0.03 & $-0.22,0.28$ & 0.12 & $-0.11,0.34$ \\
\hline Other red meat products $\ddagger \S$ & $-14 \cdot 1$ & $-18 \cdot 0,-10 \cdot 3$ & -14.4 & $-19 \cdot 2,-9 \cdot 6$ & $-13 \cdot 1$ & $-18 \cdot 3,-7 \cdot 8$ & 0.924 & -0.48 & $-0.61,-0.35$ & -0.53 & $-0.71,-0.35$ & -0.49 & $-0.68,-0.29$ \\
\hline Butter & 1.4 & $-0.3,3.2$ & $2 \cdot 1$ & $-0.5,4.8$ & 1.6 & $-0.6,3.7$ & 0.908 & 0.24 & $-0.05,0.55$ & 0.31 & $-0.07,0.72$ & 0.25 & $-0.09,0.57$ \\
\hline Whole milk & -77.7 & $-109,-46 \cdot 2$ & -63.6 & $-88.6,-38.7$ & -53.0 & $-82.1,-23.9$ & 0.513 & -0.88 & $-1.23,-0.52$ & -0.96 & $-1.34,-0.58$ & -0.93 & $-1.44,-0.42$ \\
\hline Semi-skimmed milk & 21.0 & $-6 \cdot 7,48 \cdot 8$ & $28 \cdot 7$ & $-1.9,59.2$ & $10 \cdot 9$ & $-23 \cdot 6,45 \cdot 3$ & 0.741 & 0.17 & $-0.05,0.39$ & 0.22 & $-0.01,0.46$ & 0.08 & $-0.17,0.33$ \\
\hline Skimmed milk & 6.5 & $-2 \cdot 1,15 \cdot 2$ & $12 \cdot 1$ & $4 \cdot 1,20 \cdot 1$ & $7 \cdot 2$ & $-2 \cdot 3,16 \cdot 8$ & 0.602 & 0.53 & $-0.17,1.25$ & 0.87 & $0.29,1.45$ & 0.43 & $-0.14,1.00$ \\
\hline Total milk & -53.2 & $-90.5,-15.9$ & -30.4 & $-70.8,10.0$ & $-26 \cdot 2$ & $-58 \cdot 1,5 \cdot 7$ & 0.518 & -0.21 & $-0.36,-0.06$ & -0.13 & $-0.31,0.04$ & -0.11 & $-0.25,0.02$ \\
\hline Processed potatoes & $-12 \cdot 1$ & $-16 \cdot 8,-7 \cdot 4$ & $-17 \cdot 0$ & $-21 \cdot 5,-12.5$ & $-15 \cdot 5$ & $-22 \cdot 4,-8 \cdot 6$ & 0.392 & -0.37 & $-0.52,-0.23$ & -0.61 & $-0.77,-0.45$ & -0.55 & $-0.79,-0.30$ \\
\hline Savoury snacks & $-1 \cdot 1$ & $-3 \cdot 6,1 \cdot 3$ & -1.8 & $-4.5,1.0$ & -1.9 & $-4.8,0.9$ & 0.894 & -0.08 & $-0.25,0.09$ & -0.15 & $-0.37,0.08$ & -0.15 & $-0.37,0.07$ \\
\hline Takeaway foods & $-8 \cdot 8$ & $-14 \cdot 2,-3.5$ & -11.9 & $-18 \cdot 0,-5 \cdot 9$ & -9.4 & $-16 \cdot 4,-2 \cdot 5$ & 0.688 & -0.41 & $-0.66,-0.16$ & -0.58 & $-0.88,-0.29$ & -0.47 & $-0.82,-0.12$ \\
\hline No. of households & & 1750 & & 1731 & & 1537 & & & 1750 & & 1731 & & 1537 \\
\hline No. of people & & 4022 & & 3975 & & 3371 & & & 4022 & & 3975 & & 3371 \\
\hline No. of people, weightedll & & 4935 & & 14776 & & 15356 & & & 14935 & & 14776 & & 15356 \\
\hline
\end{tabular}

SII, Slope Index of Inequality; RII, Relative Index of Inequality; SIMD, Scottish Index of Multiple Deprivation; EFS, Expenditure and Food Survey; LCFS, Living Costs and Food Survey. Household and eating out consumption combined. Significant $P$ values are indicated in bold font.

a de gradient between the most deprived and the least deprived).

From 2006 the EFS moved from a financial year to a calendar year basis. As a consequence of this the January to March 2006 data are duplicated in the $2005 / 2006$ and the 2006 results.

fMeat portion only; see appendices 2 \& 4 of Barton et al. (2012) for methodology.

burgers and pâté, and is a component of total red meat.

IThe results are weighted to the Scottish population; the number provided is approximately 1000th of the Scottish population. 
difficult to explain due to changes in consumption patterns over the three time periods. For 2001 to 2003 consumption of sugar-free drinks was highest in the least deprived, for 2004 to 2006 consumption was highest in the most deprived, and for 2007 to 2009 there was no difference.

\section{Discussion}

The current work has used an established methodology to estimate food and nutrient intakes by an area-based measure of deprivation (SIMD) from food purchase data. To our knowledge, this is the first time that household food purchase data have been analysed in relation to an area-based measure of deprivation in the UK. However Nichèle et $a l^{(32)}$ and Ricciuto and Tarasuk ${ }^{(33)}$ have used food purchase data to study socio-economic differences in food and nutrient intakes in France and Canada, respectively, and found similar disparities to those found here. In the current study statistically significant differences have been found, with foods targeted for increase (fruit and vegetables, brown/wholemeal bread, breakfast cereal, oil rich fish, white fish and complex carbohydrates) having the highest mean consumption in the least deprived quintile of the SIMD. Conversely, foods which are associated with a poorer quality diet (sugar-containing soft drinks; other red meat products (processed meat, pies and sausages); whole milk; processed potatoes; and takeaway foods) have been found to be consumed more in the most deprived quintile. Results from the earlier time periods 2001-2003 and 2004-2006 showed similar trends to those presented here, with statistically significant results being constant over the three time periods with the exception of those for potatoes, total bread, sugar and preserves, cakes and pastries, total red meat and total milk ${ }^{(31)}$. Earlier surveys showed that consumption of potatoes, sugar and preserves and total milk were significantly lower in the least deprived for 2001-2003 but no significant differences were found in later time periods. Total bread consumption was highest in the most deprived for 2001-2003 and 20072009 but not for the middle time period; consumption of cakes and pastries was only significant in 2007-2009, with greater consumption in the least deprived; and total red meat consumption was significantly higher in the most deprived quintile for the first two time periods.

Similar patterns of food consumption have been found in the Scottish Health Survey, where those in the most deprived quintile were less likely to consume the foods targeted for increase ${ }^{(34,35)}$.

The WHO guideline, Sugars intake for adults and children, has highlighted increasing concern that consumption of free sugars (especially from sugar-containing drinks) increases overall energy intake and that this may reduce the intake of more nutritionally adequate calories, which may lead to an unhealthy diet, weight gain and increased risk for non-communicable diseases ${ }^{(36)}$.
Differences found in sugar-containing soft drink consumption (higher in the most deprived groups) were reflected in a similar pattern for NMES intake. These socioeconomic differences in foods and food groups mirrored those of other recent studies. The UK Low Income Diet and Nutrition Survey (LIDNS) ${ }^{(37)}$ found that 'generally, those on low income were less likely to eat wholemeal bread and vegetables. They tended to drink more soft drinks (not diet drinks) and eat more processed meats, whole milk and sugar'. Sheehy et al. ${ }^{(38)}$ found that children living in deprived areas of Scotland were significantly less likely to consume wholemeal bread, oily fish and fruit juice and were more likely to consume sugar-containing soft drinks. In 2009, work by Armstrong and colleagues reported a strong relationship between the quality of the diet of Scottish individuals (using a Diet Quality Index) and deprivation. This relationship was strong and consistent across all ages in the population from children to the elderly ${ }^{(39)}$. A study from the USA by Grimm et al. ${ }^{(40)}$ found that a significantly lower percentage of those living with greatest poverty were likely to consume fruit at least twice daily and vegetables at least three times daily than those living with the least poverty. Turrell et al. ${ }^{(41)}$ found that those living in socio-economically disadvantaged areas of Brisbane, Australia were not associated with a tendency to purchase healthier foods compared with those living in socio-economically advantaged areas. McNeill et al. found no significant associations between total fat or saturated fat (as a percentage of food energy) and SIMD; however, those living in more deprived areas were found to consume significantly more processed meats, crisps and savoury snacks ${ }^{(42)}$. Similarly, in the present study no socio-economic differences were detected in total fat and saturated fat intake as a percentage of food energy despite the consumption of some of the main contributing foods, such as whole milk and processed meat (referred to as other red meat products in Table 2), being higher in more deprived groups. However, certain foods such as cheese and cream have not been monitored to date and these may be consumed in higher quantities in the least deprived groups, which would explain the fact that no difference was found in fat and saturated fat by SIMD. The 2001/02 NDNS found that those living in households receiving government benefits (e.g. income support) were less likely to eat cheese (women only) and cream (both genders) ${ }^{(43)}$. The exploration of foods contributing to target macronutrient intake is the subject of current work to ensure that foods targeted for change reflect current eating practices, because those targeted were based on consumption patterns from the National Food Survey of 1989 to $1991^{(44)}$. Dietary Goals for Scotland have recently been revised ${ }^{(45)}$ and now include red and processed meat, NSP and energy density, three aspects of the diet where inequalities persist.

The findings that households living in the most deprived quintiles of SIMD consumed more of the sugary and fatty 
foods monitored concurs with the finding that those living in the most deprived areas were consuming a more energy-dense diet than those living in more affluent areas ( $741 \mathrm{~kJ} / 100 \mathrm{~g}$ compared with $701 \mathrm{~kJ} / 100 \mathrm{~g}$ ). While energy intakes and percentage of energy from fat are similar across the social gradient, there is a significant difference in energy density calculated from foods and milk ${ }^{(29)}$. This may be due to less deprived households consuming a greater weight of food but with the same overall energy and fat composition (i.e. more foods with higher water content). Thus while fat intakes may not be ideal, the diets of those in the least deprived quintile are less energy dense (i.e. are consuming more fruits and vegetables) and may be more nutrient dense. Similarly the UK NDNS of 2000/01 found that those living in households not receiving benefits had significantly higher intakes of the majority of vitamins and minerals than those on benefits ${ }^{(46)}$.

The analysis by SII and RII found that absolute and relative inequalities in food/nutrient intakes have not changed appreciably between 2001 and 2009. It is difficult to explain the significant difference found between the SII values for sugar-free drink consumption, as while significant linear trends were found for consumption in 2001-2003 and 2004-2006, there was no discernible linear gradient for 2007-2009. The reason for this is unclear; however, young people are known to have the highest consumption of soft drinks and all carbonated drinks (sugared and sugar-free) have been removed from sale in schools over the period 2008-2009 ${ }^{(42)}$. Although this policy is welcomed and may contribute, it is unlikely to fully account for the population change, which may be related to the promotion of sugar-free soft drinks by the food industry or that consumption patterns are in a process of flux and change. Monitoring of future consumption patterns may help to explain these changes.

In order to be able to compare results obtained with the dietary targets/goals that are set at a population level (i.e. including non-consumers), all analyses have been conducted at the population level. However, inspection of the EFS/LCFS data suggests that the lower mean consumption found for the 'healthier foods' (e.g. wholemeal bread and oil-rich fish) in the more deprived quintiles of SIMD is due to larger numbers of non-consumers than is found in the less deprived quintiles of SIMD, rather than lower intakes by those consuming (KL Barton, unpublished results). This suggests that more emphasis needs to be placed on nonconsumers in health improvement initiatives, such as those presented in the World Cancer Research Fund's NOURISHING framework of food policies to promote healthy diets $^{(47)}$, as well as trying to get everyone to eat more of the foods targeted for increased consumption. Targeting nonconsumers could assist in reducing the socio-economic gradient found between the least and most deprived quintiles of SIMD and would also increase the population mean. Examples of health improvement initiatives include engagement with the food industry with regard to improving food labelling (EU Regulation 1169/2011) and reformulation ${ }^{(48)}$; financial incentives to the most vulnerable; and the possibility of subsidy and taxation ${ }^{(49)}$. It could be argued that improved labelling may have greater influence on the more affluent as they may be more inclined to use food labels to inform their food choices, which could lead to greater disparities between the most and least deprived (highlighting that improved labelling needs to be teamed with consumer education). Reformulation of manufactured products will affect all consumers, but may be more likely to have a greater influence on the most deprived as many of the products that are being reformulated are consumed more by the most deprived. In Scotland, the Scottish Food and Drink Federation is supporting small and medium-sized enterprises to adjust their product recipes to make them healthier through a grant provided by the Scottish Government ${ }^{(48)}$. Financial incentives to the most vulnerable include initiatives such as Healthy Start, a UK-wide government scheme (http://www.healthystart. nhs.uk/) which aims to improve the health of low-income pregnant women and families by providing vouchers for milk, plain fresh and frozen fruit and vegetables, and infant formula milk. Taxing unhealthy foods and/or subsidising healthy foods is a political issue, with the regressive effect of taxation on the poor being thought to be alleviated by subsidies of healthy foods ${ }^{(50)}$. The full effects of taxation and/or subsidies on the diet in countries that have implemented such policies have yet to be seen.

It is apparent from the results of the present study that socio-economic position is associated with dietary intake and confirms the inequality that continues to exist with regard to healthy eating. It is of particular concern that intakes of foods targeted for increased consumption are significantly lower in the most deprived groups of the population. While there is no evidence to suggest that the gap between the most and least deprived is increasing or decreasing, the improvements being seen across all quintiles of SIMD are extremely small. The work reported was commissioned specifically to inform Scottish Government policy on population (household)-level food and dietary intake, to monitor the impact of policy initiatives on secular trends in food and nutrient intakes in Scotland and to inform future policy to target diet and social inequalities. The importance of this work has been demonstrated in that it has been widely cited in Scottish policy documents, e.g. Healthy Eating, Active Living ${ }^{(51)}$ and the Review of the Scottish Diet Action Plan ${ }^{(20)}$, and it provides the data for two of the indicators for the Scottish Government's Preventing Overweight and Obesity Route Map $^{(4,52)}$. The study findings have significant implications for the design of government policy aimed at improving food and nutrient intakes in Scotland and highlight the importance of continued monitoring of dietary intake to assess the cost-effectiveness of European and national policy initiatives or action to change dietary intake, especially among more deprived communities. 


\section{Acknowledgements}

Acknowledgements: The authors would like to acknowledge the contribution of Chris Dibben, Lecturer in Geography, University of St Andrews and Anne Milne, Heather Peace and Gillian Purdon from the Food Standards Agency in Scotland. The authors would also like to thank the staff of the Department for Environment Food \& Rural Affairs (Defra), York for their invaluable help interpreting the data and formulating the calculations required for analysis of the EFS/LCFS data, in particular Jim Holding, Sarah McDiarmid and Clare Burgon; and the staff at the Office of National Statistics (ONS), Newport, Wales for their assistance in providing data on sampling variables and mapping the SIMD variable of the EFS/LCFS households. Financial support: This work was supported by the Food Standards Agency Scotland and the Scottish Government (FS424018). It was carried out using data from the UK Data Archive, University of Essex (http://www.data-archive.ac. $\mathrm{uk} /$ ); additional variables on sampling and income were provided by the ONS; and SIMD data were obtained from Scottish Neighbourhood Statistics. The findings and conclusions in this paper are those of the authors and do not necessarily represent the views of the funding agency. Conflict of interest: None. Authorship: The project was managed by W.L.W. and the Project Steering Group, which included K.L.B., W.L.W., A.S., J.A. and A.S.A. K.L.B., W.L.W., A.S., J.A. and A.S.A. designed the research; K.L.B. and W.L.W. conducted the research; K.L.B. analysed the data; K.L.B., W.L.W., A.S., J.A. and A.S.A. wrote the paper; K.L.B., W.L.W. and A.S.A. had primary responsibility for final content; all authors read and approved the final manuscript. Ethics of human subject participation: Ethical approval was not required.

\section{References}

1. Barnett K, Mercer SW, Norbury M et al. (2012) Epidemiology of multimorbidity and implications for health care, research, and medical education: a cross-sectional study. Lancet $\mathbf{3 8 0}$, $37-43$.

2. House of Commons Select Committee on Health (2004) Third Report of Session 2003-04: Obesity. London: The Stationery Office.

3. Lim SS, Vos T, Flaxman AD et al. (2012) A comparative risk assessment of burden of disease and injury attributable to 67 risk factors and risk factor clusters in 21 regions, 1990-2010: a systematic analysis for the Global Burden of Disease Study 2010. Lancet 380, 2224-2260.

4. Scottish Government (2010) Preventing Overweight and Obesity in Scotland: A Route Map Towards Healthy Weight. Edinburgh: The Scottish Government.

5. Scarborough P, Bhatnagar P, Wickramasinghe KK et al. (2011) The economic burden of ill health due to diet, physical inactivity, smoking, alcohol and obesity in the UK: an update to 2006-07 NHS costs. J Public Health (Oxf) 33, 527-535.

6. Department of Health and Social Security (1980) Inequalities in Health: Report of a Research Working Group. London: DHSS.
7. Department of Health (1998) Independent Inquiry into Inequalities in Health (The Acheson Report). London: Department of Health.

8. Commission on Social Determinants of Health (2008) Closing the Gap in a Generation: Health Equity Through Action on the Social Determinants of Health. Final Report of the Commission on Social Determinants of Health. Geneva: WHO.

9. Department of Health (2010) Fair Society, Healthy Lives (The Marmot Review). London: Department of Health.

10. Marmot M \& Wilkinson RG (2006) Social Determinants of Health, 2nd ed. Oxford: Oxford University Press.

11. Darmon N \& Drewnowski A (2008) Does social class predict diet quality? Am J Clin Nutr 87, 1107-1117.

12. Marmot M (2013) Review of Social Determinants and the Health Divide in the WHO European Region: Final Report. London: UCL Institute of Health Equity and WHO.

13. Audit Scotland (2012) Health Inequalities in Scotland. Edinburgh: Audit Scotland.

14. Scottish Government (2008) Equally Well Report of the Ministerial Task Force on Health Inequalities. Edinburgh: The Scottish Government.

15. Scottish Government (2008) The Early Years Framework. Edinburgh: The Scottish Government.

16. Scottish Government (2008) Achieving Our Potential: A Framework to Tackle Poverty and Income Inequality in Scotland. Edinburgh: The Scottish Government.

17. Scottish Government (2010) Equally Well Review 2010: Report by the Ministerial Task Force on implementing Equally Well, the Early Years Framework and Achieving Our Potential. Edinburgh: The Scottish Government.

18. The Scottish Office (1996) Scotland's Health a Challenge to us All-Eating for Health: A Diet Action Plan for Scotland. Edinburgh: The Scottish Office Department of Health.

19. The Scottish Office (1993) Scotland's Health a Challenge to us All. The Scottish Diet: Report of a Working Party to the Chief Medical Officer for Scotland. Edinburgh: The Scottish Office Home and Health Department.

20. Lang T, Dowler E \& Hunter DJ (2006) Review of the Scottish Diet Action Plan: Progress and Impacts 1996-2005. Edinburgh: Health Scotland.

21. Kirkpatrick SI, Dodd KW, Reedy J et al. (2012) Income and race/ethnicity are associated with adherence to food-based dietary guidance among US adults and children. J Acad Nutr Diet 112, 624-635.e6.

22. Wrieden WL, Bolton-Smith C, Brown CA et al. (1993) Fruit and vegetable consumption in North Glasgow: some results from the MONICA study of 1986 and 1989. Proc Nutr Soc 52, $12 \mathrm{~A}$.

23. Harrington J, Fitzgerald AP, Layte R et al. (2011) Sociodemographic, health and lifestyle predictors of poor diets. Public Health Nutr 14, 2166-2175.

24. McLennan D, Barnes H, Noble M et al. (2011) The English Indices of Deprivation 2010. London: Department for Communities and Local Government.

25. Scottish Government (2012) Scottish Index of Multiple Deprivation. http://www.scotland.gov.uk/Topics/Statistics/ SIMD/ (accessed December 2013).

26. Scottish Public Health Observatory (2007) Measuring SocioEconomic Inequalities in Health: A Practical Guide. Edinburgh: ScotPHO.

27. Wrieden WL, Armstrong J, Sherriff A et al. (2013) Slow pace of dietary change in Scotland: 2001-9. Br J Nutr 109, 1892-1902.

28. Department for Environment Food \& Rural Affairs (2012) Family Food. http://www.defra.gov.uk/statistics/foodfarm/ food/familyfood/ (accessed December 2012).

29. Wrieden WL, Armstrong J, Anderson AS et al. (2014) Choosing the best method to estimate the energy density of a population using food purchase data. J Hum Nutr Diet (Epublication ahead of print version). 
30. Shaw M, Galobardes B, Lawlor D et al. (2007) The Handbook of Inequality and Socioeconomic Position: Concepts and Measures. Bristol: Policy Press.

31. Barton KL \& Wrieden WL (2012) Estimation of food and nutrient intakes from food survey data in Scotland 2001-2009. http://www.foodbase.org.uk//admintools/reportdocuments/ 749-1-1324_Final_Report_2001-2009.pdf (accessed December 2013).

32. Nichèle V, Andrieu E, Boizot C et al. (2005) La consommation d'aliments et de nutriments en France: évolution 1969-2001 et déterminants socio-économiques des comportements (Food and Nutrient Intakes in France: 1969-2001 Trends and Socioeconomic Determinants). Ivry-sur-Seine: Laboratoire de Recherche sur la Consommation. (in French).

33. Ricciuto LE \& Tarasuk VS (2007) An examination of incomerelated disparities in the nutritional quality of food selections among Canadian households from 1986-2001. Soc Sci Med 64, 186-198.

34. Bromley C, Chaudhury M, Deverill C et al. (2005) The Scottish Health Survey 2003. vol. 2: Adults. Edinburgh: The Scottish Executive.

35. Bromley C, Corbett J, Day J et al. (2011) Scottish Health Survey 2010. vol. 1: Main Report. Edinburgh: The Scottish Government.

36. World Health Organization (2013) Draft Guidelines on Free Sugars Released for Public Consultation. Geneva: WHO.

37. Nelson M, Erens B, Bates B et al. (2007) Low Income, Diet and Nutrition Survey. London: The Stationery Office.

38. Sheehy C, McNeill G, Masson L et al. (2008) Survey of sugar intake among children in Scotland. http://www.food.gov. uk/multimedia/pdfs/sugarintakescot2008rep.pdf (accessed June 2013)

39. Armstrong J, Sherriff A, Wrieden WL et al. (2009) Deriving and Interpreting Dietary Patterns in the Scottish Diet: Further Analysis of the Scottish Health Survey and the Expenditure and Food Survey. Aberdeen: Food Standards Agency Scotland.

40. Grimm KA, Foltz JL, Blanck HM et al. (2012) Household income disparities in fruit and vegetable consumption by state and territory: results of the 2009 behavioral risk factor surveillance system. J Acad Nutr Diet 112, 2014-2021.

41. Turrell G, Blakely T, Patterson C et al. (2004) A multilevel analysis of socioeconomic (small area) differences in household food purchasing behaviour. J Epidemiol Community Health 58, 208-215.
42. McNeill G, Masson LF, Craig LCA et al. (2010) Sugar and fat intake among children in Scotland: what is needed to reach the dietary targets? Public Health Nutr 13, 1286-1294.

43. Henderson L, Gregory J \& Swan G (2002) The National Diet and Nutrition Survey: Adults Aged 19-64 Years. vol. 1: Types and Quantities of Foods Consumed. London: The Stationery Office.

44. Department for Environment Food \& Rural Affairs (2001) National Food Survey 2000. London: The Stationery Office.

45. The Scottish Government (2013) Revised Dietary Goals for Scotland. http://www.scotland.gov.uk/Topics/Health/HealthyLiving/Food-Health/DietaryGoalsScot (accessed June 2013).

46. Henderson L, Irving K, Gregory J et al. (2003) The National Diet and Nutrition Survey: Adults Aged 19-64 Years. vol. 3: Vitamin and Mineral Intakes and Urinary Analytes. London: The Stationery Office.

47. Hawkes C, Jewell J \& Allen K (2013) A food policy package for healthy diets and the prevention of obesity and dietrelated non-communicable diseases: the NOURISHING framework. Obes Rev 14, 159-168.

48. Scottish Food and Drink Federation (2011) SFDF Reformulation Programme. http://www.sfdf.org.uk/sfdf/priorities_ reformulation.aspx (accessed May 2014).

49. Mytton OT, Clarke D \& Rayner M (2012) Taxing unhealthy food and drinks to improve health. BMJ 344, e2931.

50. Nnoaham KE, Sacks G, Rayner M et al. (2009) Modelling income group differences in the health and economic impacts of targeted food taxes and subsidies. Int J Epidemiol 38, 1324-1333.

51. Scottish Government (2008) Healthy Eating, Active Living: An Action Plan to Improve Diet, Increase Physical Activity and Tackle Obesity (2008-2011). Edinburgh: The Scottish Government.

52. Scottish Government (2012) Obesity Indicators (2012) Monitoring Progress for the Prevention of Obesity Route Map. Edinburgh: The Scottish Government.

53. Department of Health (1991) Dietary Reference Values for Food Energy and Nutrients for the United Kingdom: Report of the Panel on Dietary Reference Values of the Committee on Medical Aspects of Food Policy. Report on Health and Social Subjects no. 41. London: HMSO.

54. Wrieden WL, Barton KL, Armstrong J et al. (2006) A review of food consumption and nutrient intakes from national surveys in Scotland: comparison to the Scottish Dietary Targets. http://www.food.gov.uk/multimedia/pdfs/scotdietrytarg.pdf (accessed December 2013). 